\title{
Autosomal Recessive Spastic Ataxia of Charlevoix-Saguenay
}

National Cancer Institute

\section{Source}

National Cancer Institute. Autosomal Recessive Spastic Ataxia of Charlevoix-Saguenay. NCI Thesaurus. Code C154614.

An autosomal recessive condition caused by mutation(s) in the SACS gene, encoding sacsin. It is characterized by early onset cerebellar ataxia, pyramidal tract signs and peripheral neuropathy. 